\title{
The optical and X-ray flickering of XTE J1118+480
}

\author{
J. Malzac ${ }^{1,2}$, T. Belloni ${ }^{2}$, H. C. Spruit ${ }^{3}$, and G. Kanbach ${ }^{4}$ \\ 1 Institute of Astronomy, Madingley Road, Cambridge, CB3 0HA, UK \\ 2 Osservatorio Astronomico di Brera, Via Brera 28, 20121 Milano, Italy \\ 3 Max-Planck-Institut für Astrophysik, Postfach 1317, 85741 Garching, Germany \\ ${ }^{4}$ Max-Planck-Institut für Extraterrestrische Physik, Postfach 1317, 85741 Garching, Germany
}

Received 21 January 2003 / Accepted 27 May 2003

\begin{abstract}
We use both time-domain and Fourier techniques to study the correlated optical/X-rays variability in the black hole $\mathrm{X}$-ray nova XTE J1118+480. Its X-ray timing properties such as the shape of the X-ray power spectrum and cross-correlation functions (CCFs) between X-ray energy bands, the slight decrease of rms variability from $30 \%$ in the $2-5.9 \mathrm{keV}$ to $19 \%$ in the 15.5-44.4 keV band, as well as the X-ray hardness/flux anti-correlation are very similar to what is found in other black hole binaries in the hard state. The optical/X-ray CCF is virtually independent of the X-ray energies. The optical flux appears to be correlated not only with the X-ray flux but also with the X-ray spectral variability. Both the coherence function and the lags between optical and the X-rays are Fourier frequency dependent. The optical/X-ray coherence function reaches its maximum $(\sim 0.3)$ in the $0.1-1 \mathrm{~Hz}$ range and the time-lags decrease with frequency approximatively like $f^{-0.8}$. The correlation between X-ray and optical light curves appears to have time-scale-invariant properties. The X-ray/optical CCF maintains a similar but rescaled shape on time-scales ranging at least from $0.1 \mathrm{~s}$ to few $10 \mathrm{~s}$. Using the event superposition method we show that the correlation is not triggered by a single type of event (dip or flare) in the light curves. Instead, optical and X-ray fluctuations of very different shapes, amplitudes and time-scales are correlated in a similar mode where the optical light curve is seemingly related to the time derivative of the X-rays.
\end{abstract}

Key words. black hole physics - stars: individual: V*KV UMa - X-rays: binaries - X-rays: individuals: XTE J1118+480

\section{Introduction}

The X-ray nova XTE J1118+480 $\left(=\mathrm{V}^{*} \mathrm{KV} \mathrm{UMa}\right)$, was discovered by the Rossi X-Ray Timing Explorer (RXTE) All-Sky Monitor (ASM) on March 292000 (Remillard et al. 2000) as a slowly rising source. The outburst lasted a few months with a plateau-like X-ray light-curve that rapidly fell down around mid-July. A post-analysis revealed an an earlier outburst in January 2000. The optical spectrophotometry proved a low mass X-ray binary system containing a black hole of at least $6 M_{\odot}$ (Mc Clintock et al. 2001a; Dubus et al. 2001; Wagner et al. 2001). The interstellar extinction towards the source is exceptionally low (Garcia et al. 2000). This fact allowed an unprecedented wavelength coverage (Mauche et al. 2000; Hynes et al. 2000; McClintock et al. 2001b; Hynes et al. 2003; Chaty et al. 2003 and reference therein). In the radio to optical bands, a strong non-thermal component was associated with synchrotron emission from a powerful jet or outflow (Fender et al. 2001). In the optical to EUV bands the spectral energy distribution is dominated by a thermal component from the accretion disc. The X-ray emission consists in a typical powerlaw spectrum with photon index $\Gamma \sim 1.8$. Such a spectrum is generally associated with Comptonisation in

Send offprint requests to: J. Malzac,

e-mail:malzac@ast.cam.ac.uk the hot inner part of the disc or corona. In the case of XTE J1118+480, alternative interpretations in terms of jet emission through inverse Compton (Georganopoulos et al. 2002) or synchrotron (Markoff et al. 2001) were also put forward.

The X-ray variability is characterized by a flaring activity on time-scales of a few seconds typical of accreting black hole sources (Revnivtsev et al. 2000). A $\sim 0.1 \mathrm{~Hz}$ quasi-periodic oscillation (QPO) was reported by the same authors and subsequently confirmed by the ASCA data (Yamaoka et al. 2000) as well as other RXTE observations where it appeared to be variable (Wood et al. 2000). The simultaneous RXTE/HST observations showed that a similar QPO is also present in the $H S T$ data and that the X-rays and optical/UV bands are correlated on time-scales of seconds (Haswell et al. 2000; Hynes et al. 2003).

Kanbach et al. (2001, hereafter K01) performed 4 observations on the nights of 4, 5, 6, and 7 July 2000, shortly before the end of the outburst. They combined simultaneous observations with RXTE and the rapid optical photo-meter OPTIMA (Straubmeier et al. 2001) attached to the $1.3 \mathrm{~m}$ telescope on Mt Skinakas, Crete. They studied the X-ray and optical auto-correlation functions (ACFs) and X-ray/optical cross-correlation (CCFs). They found the optical ACF to be significantly narrower than the X-ray ACF. The full width at 
mid height (FWMH) of the two ACFs differs by a factor $>2$. They found the correlation between the optical and X-ray light curves to be surprisingly complex. The CCF rises very quickly at positive optical lags, peaks around $0.5 \mathrm{~s}$ and then declines slowly at larger lags. Therefore, the optical light curve tends to be delayed relatively to the $\mathrm{X}$-rays by a relatively long time (of order of $1 \mathrm{~s}$ ). Strikingly, the two bands appear to be anticorrelated at negative optical lags indicating a systematic optical dip 1-2 s before the X-rays reach their maximum. As this feature suggests that the optical band "knows" about what will happen in the X-rays about $1 \mathrm{~s}$ later, it was named "precognition dip" by K01. K01 further showed that the detailed shape of the CCF is variable on time-scales as short as 30 s. Spruit \& Kanbach (2002, hereafter SK02) analyzed this variability of the $\mathrm{CCF}$ in terms of a principal component analysis. They found that there are two systematic components which vary statistically independently. Both have the shape of a broad dip followed by a sharper peak, but their duration differs by a factor of about 3 .

K01 argued that those characteristics rule out reprocessing as the origin of the optical variability and favors synchrotron emission. Merloni et al. (2000) had previously interpreted the correlated X-ray/optical variability in terms of thermal Comptonisation of synchrotron radiation in an accretion disc corona. It is not clear however how such a model could reproduce the complicated shape of the cross-correlation function. K01 proposed a somewhat different scenario where the $\mathrm{X}$-rays are produced within a few hundred Schwarzschild radii from the black hole and the optical is produced by synchrotron effect at much larger distance, in a magnetic outflow. In this framework the optical lags would be due to the travel-time of a perturbation from the disc to the optical photo-sphere of the outflow. This scenario however (as well as reprocessing models) does not explain the "precognition dip".

The only other source for which simultaneous X-optical observations at millisecond time resolution have been reported is GX 339-4, where an anti-correlation between the X-rays and optical, similar to the "precognition" dip, was observed (Motch et al. 1982). Fabian et al. (1982) interpreted the optical emission as cyclo-synchrotron radiation produced in the inner parts of the accretion flow.

In this paper we use the data of K01 to investigate further the optical X-ray correlation in XTE J1118+480. The optical observations and data processing are described in K01 and SK02. The PCA X-ray light curves used in these previous analyses were decomposed into 3 energy bands: 2$5.9 \mathrm{keV}$ (referred hereafter as b1), 5.9-15.5 keV (b2) and $15.5-44.4 \mathrm{keV}$ (b3). In order to study a range of time-scales, we used only the data for which we had a sufficiently long continuous exposure. The analysis was performed over 11 light curves segments of $590 \mathrm{~s}$ duration and $3 \mathrm{~ms}$ resolution taken during the four observation nights. Most of the results shown in this paper are averaged over all these data segments. The paper is divided into three sections presenting successively the results from the time-domain, Fourier domain and superposition analysis. These results are then summarized and briefly discussed in Sect. 5.

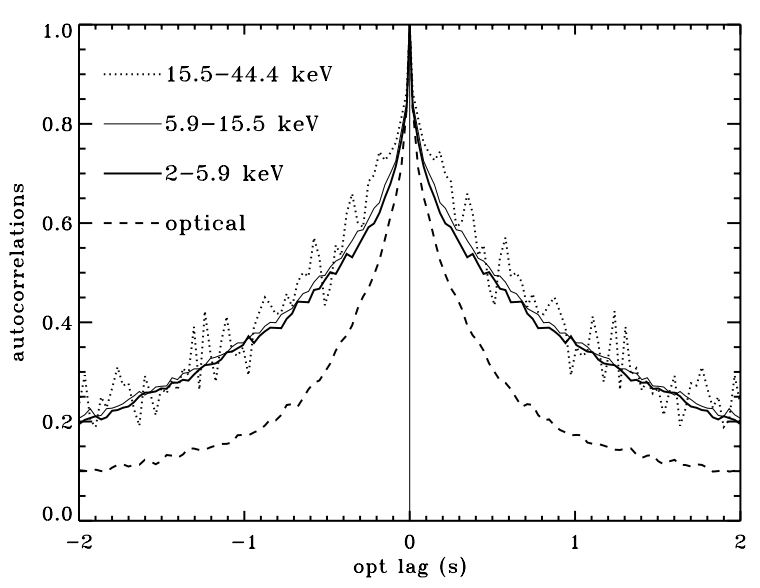

Fig. 1. Optical and X-ray ACFs of the photon flux in the X-ray bands b1, b2, b3 and optical. In this figure as well as in Figs. 2 and 3, the low frequency noise on time-scales longer than $100 \mathrm{~s}$ was removed by dividing the light curves by the piecewise linear trend. This piecewise linear trend is defined as the linear interpolation between the $100 \mathrm{~s}$ average count rate computed at time bins separated by $50 \mathrm{~s}$. The presence of noise due to the optical telescope oscillations (see SK02 and Sect. 3.2) significantly affects the shape of the ACF. Therefore, only the 2 segments in which these oscillations are minimal were used to estimate the optical ACF. For clarity, the ACFs were rebinned with $30 \mathrm{~ms}$ resolution.

\section{Time domain analysis}

\subsection{X-ray properties}

We first examine the properties of the X-ray light curves in the time domain. The X-ray ACFs in the three energy bands are, within the uncertainties, indistinguishable from the total X-ray ACF and broader than the optical ACF (as shown in Fig. 1). The FWMH of the X-ray CCFs is $\sim 1 \mathrm{~s}$ while that of the optical is only of $0.4 \mathrm{~s}$. The X-ray CCFs of the different energy bands, shown in the panels (a) and (b) of Fig. 2, are all strongly peaked at lags $<30 \mathrm{~ms}$ with a shape very similar to that of the ACFs. The different energy bands are thus strongly correlated at (nearly) 0 lag. These results are very similar to that of Maccarone et al. (2000) for Cygnus X-1.

We then searched for correlations between variations of the $\mathrm{X}$-ray hardness and the $\mathrm{X}$-ray flux. We computed the ratio of the two light curves in two energy bands b3 and b1. The resulting time series represents the time dependent hardness $b 3 / b 1$. Then we computed its CCF with the third energy band b2. In practice, the hardness ratio $b 3 / b 1$ cannot be computed for the b1 time bins containing 0 counts. We thus rebinned the light curves with a $90 \mathrm{~ms}$ resolution before estimating b3/b1, so that zero count bins are reduced to a negligible fraction of the total number of bins. For the few remaining bins with zero count, b3/b1 was arbitrarily set to the time averaged hardness ratio.

Panel of (c) of Fig. 2 displays the resulting hardness vs. flux CCF. Clearly the count rate in the b2 band is strongly anti-correlated with hardness at (almost) zero lags. The source is thus softer when brighter for fluctuations of time-scale of seconds. We note also that this CCF shape is similar to the shape of the X-ray ACFs, suggesting that spectral variations map very closely the flux. We also computed the hardness-flux 

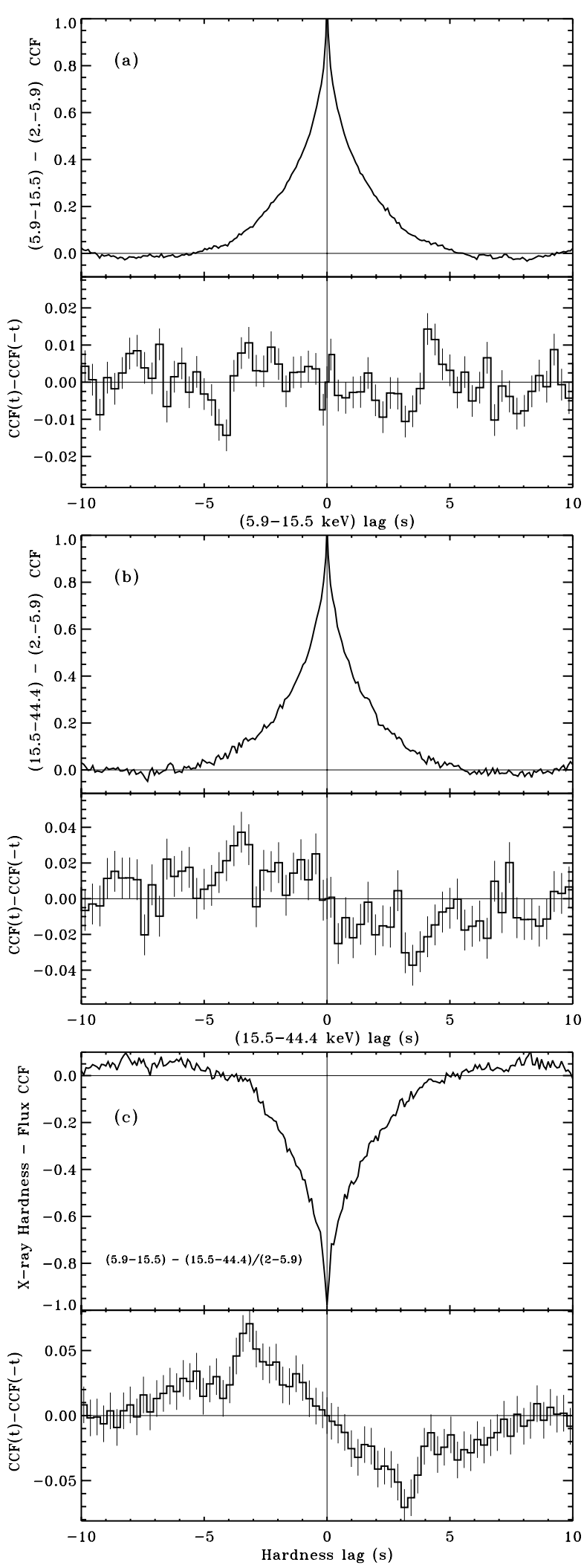

Fig. 2. X-ray CCFs and their anti-symmetric parts $C C F(t)-C C F(-t)$. a) flux in band b2 vs. flux in band b1. b) flux in band b3 vs. flux in band b1. c) hardness ratio b3/b1 vs. flux in band b2. The one sigma uncertainties on the anti-symmetrized CCFs were estimated from the photon noise in the original data using the standard statistical error propagation method. The CCFs and anti-symmetrized CCFs were rebinned with $90 \mathrm{~ms}$ and $300 \mathrm{~ms}$ resolution respectively. correlation for the different combinations between our three energy bands and found very similar results. The b2/b1-b3 CCF appeared noisier indicating that the amplitude of the fluctuations of the $b 2 / b 1$ hardness is lower than that of $b 3 / b 1$ or $b 3 / b 2$. Such a hardness/flux anti-correlation looks similar to what found in Cygnus X-1 in the hard state (see e.g. Li et al. 1999; Feng et al. 1999)

The antisymmetrized CCFs (i.e. $\mathrm{CCF}(\mathrm{t})-\mathrm{CCF}(-\mathrm{t})$ ), also shown in Fig. 2, indicate that the X-ray CCFs as well as the hardness flux CCF are asymmetric for lags in the approximate range $1-5 \mathrm{~s}$ (outside this range the poor statistics in the XTE J1118+480 data did not enable us to firmly establish any asymmetry). The shape of the asymmetries suggests that the spectrum is relatively harder during the rising phase of a flare than its decay. In term of lags between X-ray bands this implies that the soft band is delayed with respect to the hard one. This kind of asymmetry is thus opposite to that found by Maccaronne et al. (2000) in the X-ray CCFs of Cygnus X-1, and more generally to the hard lags often reported in hard-state sources.

\subsection{Optical/X-ray correlations}

We then investigated the dependence of the optical/X-ray correlation on the X-ray energies. We found that the optical/X-ray $\mathrm{CCF}$ does not depend significantly on the X-ray band considered. Actually, our 3 optical/X-ray CCF are identical within the uncertainties, as shown in Fig. 3a.

The optical/X-ray hardness CCF (shown in Fig. 3b) is qualitatively similar to the optical/X-ray $f u x$ CCFs modulo a mirror symmetry around the X-ray axis. The optical flux and X-ray hardness are indeed anti-correlated at positive optical lags and positively correlated at negative lags. This feature derives naturally from the X-ray flux hardness anti-correlation demonstrated in the previous section. It follows that the optical flux is correlated not only with the X-rays count rate but also with the X-ray spectral variability. Similar results were found for the hardness ratios computed with the different combinations of the 3 bands.

The time-domain analysis presented above provides only information (such as time lags) that is averaged over all timesscales present in the signal. However, in general, the properties of time-series or the correlation between two time-series does depend on the time-scale considered. In order to study the dependence of the optical/ X-ray CCF on the time-scale of the fluctuations we thus filtered the light curves before computing the CCF keeping only a small range of time-scales in both signals.

The results are displayed in Fig. 4. For all of the different time-scales considered, both the anti-correlation at negative lags and positive correlation at positive lags is present. The characteristics lags appears to depend on the time scale of the fluctuations. The lag of the optical peak increases from $\sim 30 \mathrm{~ms}$ for time-scales shorter than $0.1 \mathrm{~s}$ to $\sim 3 \mathrm{~s}$ for the $10-100 \mathrm{~s}$ fluctuations. Similarly the optical dip ranges from $\sim-6 \mathrm{~ms}$ to $\sim-30 \mathrm{~s}$, so that, in the rescaled plot, we see both the optical peak and dip shifting toward negative lags as the time-scale of the 

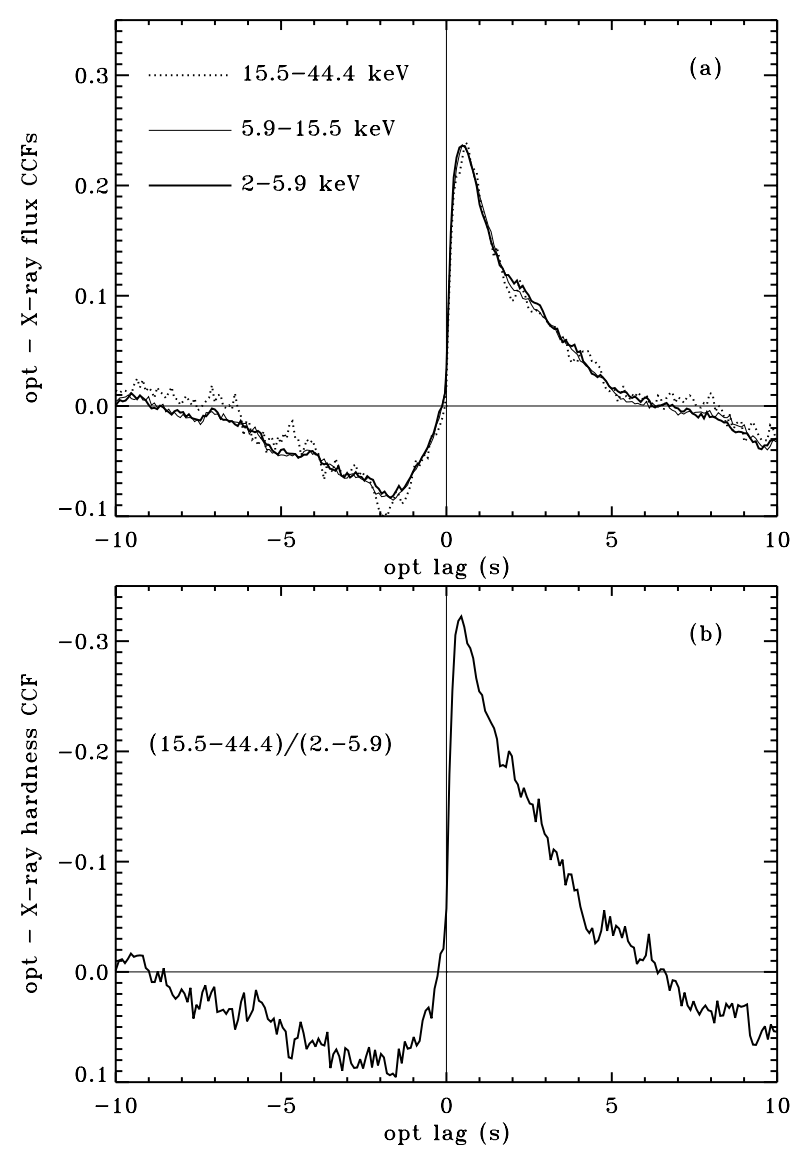

Fig. 3. Correlations between X-ray and the optical. and X-ray flux. a) X-ray flux vs. optical flux CCF for the $3 \mathrm{X}$-ray bands. b) X-ray hardness vs. optical flux CCF. For clarity the CCFs were rebinned with a 90 ms resolution.

fluctuations increases. Still in the rescaled plot, the optical flare appears narrower at longer fluctuations.

Despite these significant differences, the overall shape of the CCF appears similar but rescaled over a range covering more than three decades of time-scales.

\section{Fourier analysis}

In the following, we will use a Fourier analysis to explore more quantitatively the dependence of the optical/X-ray correlation in XTE J1118+480 on the time-scale of the fluctuations.

\subsection{Power density spectra}

Figure 5a shows the X-ray and optical power density spectra (PDS). The PDS were computed, averaged, rebinned and normalized to the total rms variability as described in Nowak et al. (1999). The counting noise was approximated by a white noise component that was then subtracted from the total PDS.

The fractional rms variability amplitude integrated over the frequency range $10^{-3}-10^{2} \mathrm{~Hz}$ is about $26 \%$ in the X-rays and $13 \%$ in the optical. The optical and total X-ray power spectra were already shown and discussed in SK02. Here we will just note the similarities of the X-ray PDS with that of other black hole candidates in the hard state such as the plateau like

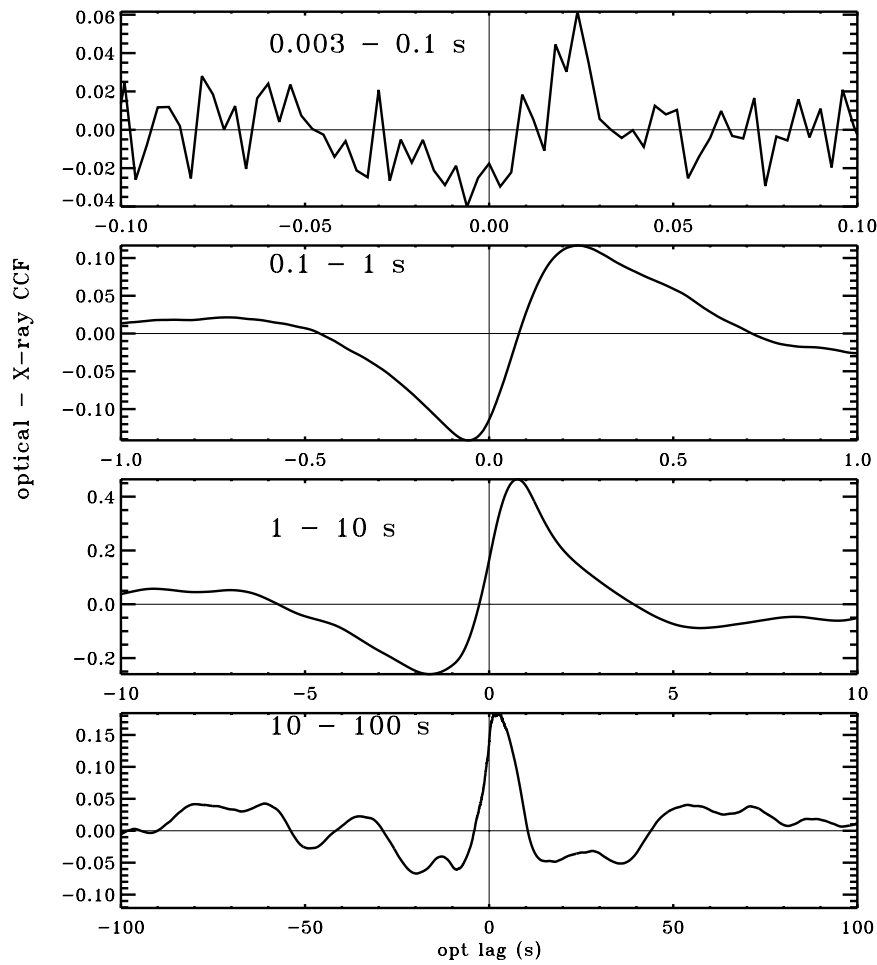

Fig. 4. Optical vs. X-ray CCFs for different time-scales of the fluctuations. The CCFs were computed after both light curve have been filtered keeping only the time-scales in the range indicated. High frequency noise was removed applying a box car filter, the low frequency noise was removed by dividing the light curve by the piecewise linear trend.

shape up to $\sim 0.1 \mathrm{~Hz}$ and then a power law component. We note also that in our July observation the X-ray PDS is slightly different from that found in the earlier observation of Revnivtsev et al. (2000) with a break at higher frequencies $(0.1 \mathrm{~Hz}$ instead of $0.03 \mathrm{~Hz}$ ) a flatter power-law component with slope $\sim 1.4$ as compared to the 1.6 slope reported by Revnivtsev et al. 2000 . This evolution of the power spectrum was accompanied by a significant reduction of the total rms amplitude that was $40 \%$ during the observation of Revnivtsev et al. More strikingly, the power spectra of Fig. 5a show little to no evidence for the QPO reported in previous observations both in the X-ray and optical (see e.g. for comparison Fig. 1 of Revnivtsev et al. 2000). This is however consistent with the previously reported trend in the evolution of the timing properties of the source during the outburst. Indeed the RXTE monitoring campaign showed that all the characteristics frequencies increased with time and the QPO became much weaker (Wood et al. 2000; Hynes et al. in prep.).

As shown in Fig. 6, the X-ray PDS depends only slightly on energy. We find that the PDS in the two lower energy bands (b1 and b2) are extremely similar. On the other hand, at higher energy (b3) the shape of the PDS differs significantly at low frequency and the integrated rms amplitude is lower (19\% instead of $\sim 30 \%$ in b1 and b2). A similar energy dependence of the rms variability amplitude is reported in Cygnus X-1 in the hard state (e.g. Nowak et al. 1999). 


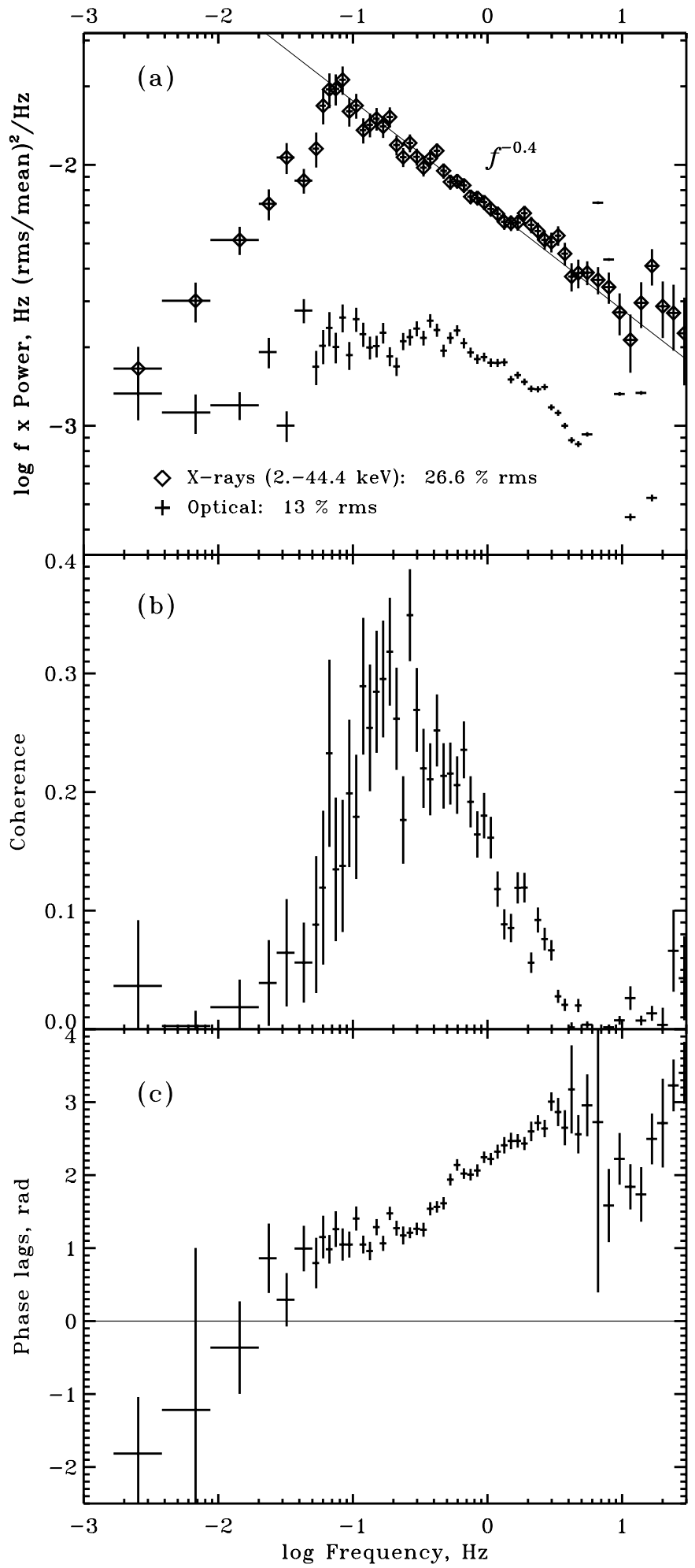

Fig. 5. The optical/X-ray correlation in the Fourier domain. a) X-ray and optical power spectra. The counting noise was subtracted (see Sect. 3.1). b) X-ray/optical coherence. c) phase-lags as function of Fourier frequency. A positive lag implies that the optical is delayed with respect to the X-rays. Uncertainties where computed according to Eq. (8) of Vaughan \& Nowak (1997) for the coherence, and Eq. (16) of Nowak et al. (1999) for the phase-lags. All error bars are at the 1 sigma level.

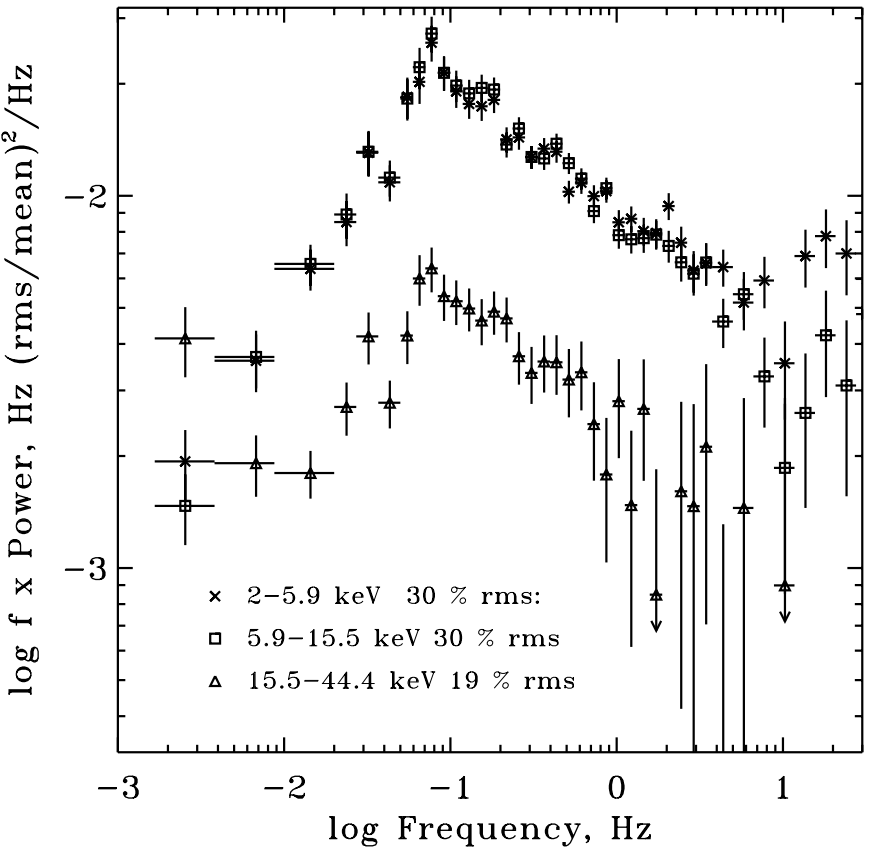

Fig. 6. X-ray power spectra in bands b1, b2 and b3. The counting noise was subtracted (see Sect. 3.1).

\subsection{Coherence and lags}

The coherence function represents the degree of linear correlation between two time series as a function of Fourier frequency (Vaughan \& Nowak 1997). The coherence function between different X-ray bands appears to be unity over a wide range of frequencies in black hole candidates (Nowak et al. 1999). We found a similar behaviour in XTE J1118+480 as shown in Fig. 7a.

The X-ray phase lags between energy bands are shown in Fig. 7b. Due to the poor statistics of the data, the phase lags are very difficult to measure. In order to improve the statistic we averaged the frequency bins over a broad range of frequencies (nearly a decade). Even so, we get essentially upper limits that are above the typical amplitude of the hard lags reported in Cyg X-1 or GX 339-4 in the hard state. There is however an indication that the lags are increasing with frequency, and surprisingly, the lags tend to be negative at low frequency. In particular in the frequency range $2 \times 10^{-3}-2 \times 10^{-2} \mathrm{~Hz}$, the phase lag between $\mathrm{b} 3$ and $\mathrm{b} 1$, is detected at the 3 sigma level and is negative. This implies that the soft band leads the hard band by 2 to $20 \mathrm{~s}$ in this frequency range. This is qualitatively consistent with the asymmetry found in the X-ray CCFs (see Sect. 2.1). This result should however be regarded with caution since it involves frequencies that correspond to time scales approaching the duration of the light-curve segments. In an attempt to improve the statistics, we used public RXTE archive data to compute the phase-lag spectra with a longer exposure time. These additional data consisted in a set of observations made between April 15 and May 23, 2000 with a total exposure time of $33 \mathrm{ks}$. Unfortunately, although we found a similar trend for negative lags at low frequencies, the results were still inconclusive. 

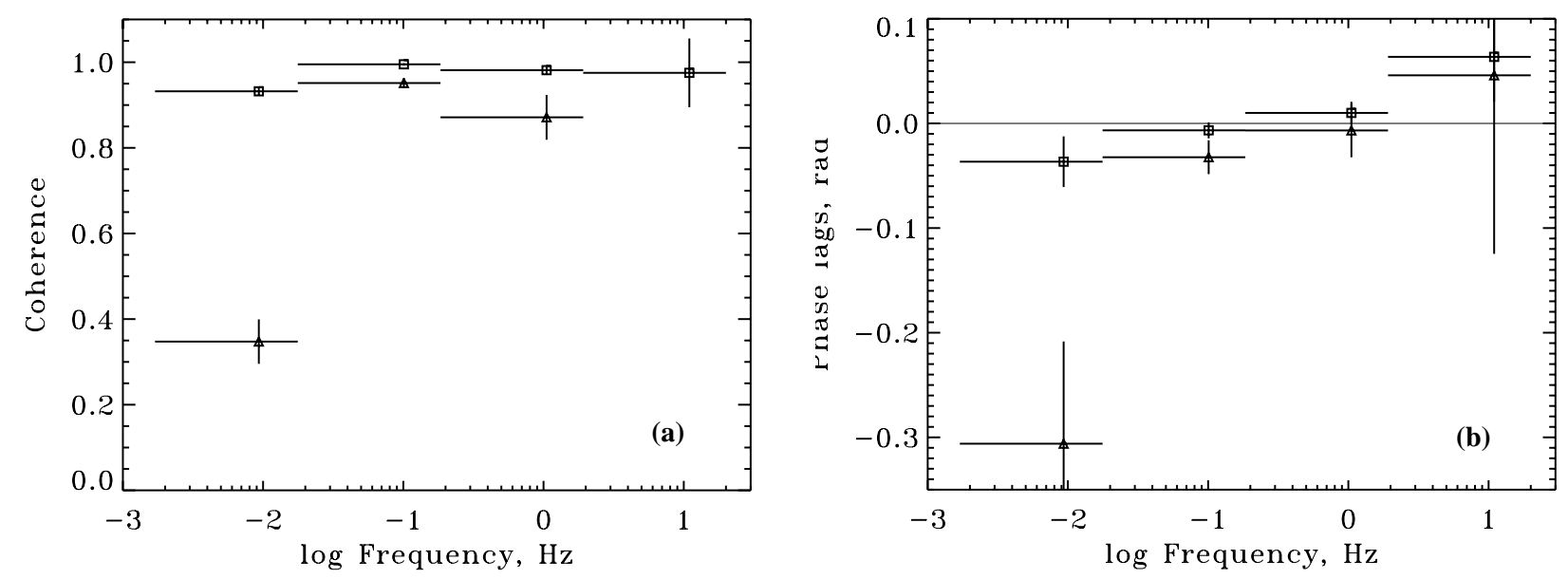

Fig. 7. a) X-ray coherence and b) phase lags. Squares stand for b2 versus b1, triangles for b3 vs. b1. Due to the high noise level it has not been possible to estimate conveniently the b3 vs. b1 coherence in the higher frequency bin.

On the other hand, the data enable us to clearly detect the optical light curve lagging the X-ray one. The optical phase lags as a function of Fourier frequency are displayed in Fig. 5c. These phase-lags increase significantly with the Fourier frequency covering a broad range of angles centered around $\pi / 2$. This leads to time lags (shown in Fig. 8) decreasing with frequency approximately like $f^{-0.8}$ in the range $10^{-2}-10 \mathrm{~Hz}$ and spanning roughly $10^{-2}-10 \mathrm{~s}$ over this frequency range. The frequency dependence of the lags is not exactly a power law as additional structures are apparent, in particular around $0.5 \mathrm{~Hz}$. Such structures are even more apparent in the phase lag diagram. Interestingly, as in the case of X-ray lags the data suggest the presence of negative lags at low frequencies. The significance of these negative lags is however very low.

The optical vs. X-ray coherence function (shown in Fig. 5b) is frequency dependent as well. It reaches a maximum in the $0.1-1 \mathrm{~Hz}$ range and decreases rapidly both at lower and higher frequency. The overall coherence is not very high $(<0.3)$ indicating that only a relatively small fraction of the optical and $\mathrm{X}$-ray variability are actually correlated. As the coherence gives only the relative importance of correlated variability, it is not clear whether the observed frequency dependence is a property of of the physical process producing the correlation, or if it is driven mainly by changes in the amplitude of the uncorrelated fraction of the variability. We note that part of the loss of coherence around $10 \mathrm{~Hz}$ is due to the artifact QPO visible in the optical power spectrum that is due to telescope oscillations (see Spruit \& Kanbach 2002).

This Fourier analysis is consistent with the CCF shape. Basically the CCF plots the coherence as a function of timelags weighted with the fractional amplitudes of variability at the frequencies producing such lags. The CCF indeed appears to peak at values $\sim 0.3$ at lags $\sim 0.5$ s i.e. roughly corresponding to the frequency range where coherence is maximum.

In addition, the reduction of the optical time-lags with frequency while the phase-lags increase is qualitatively consistent with the changes of the optical peak lag in the $\mathrm{CCF}$ at different time-scales of the fluctuations (shown in Fig. 4 and discussed in Sect. 2.2). On the other hand the Fourier time-lag diagram does not show any evidence for the optical dip at negative lags

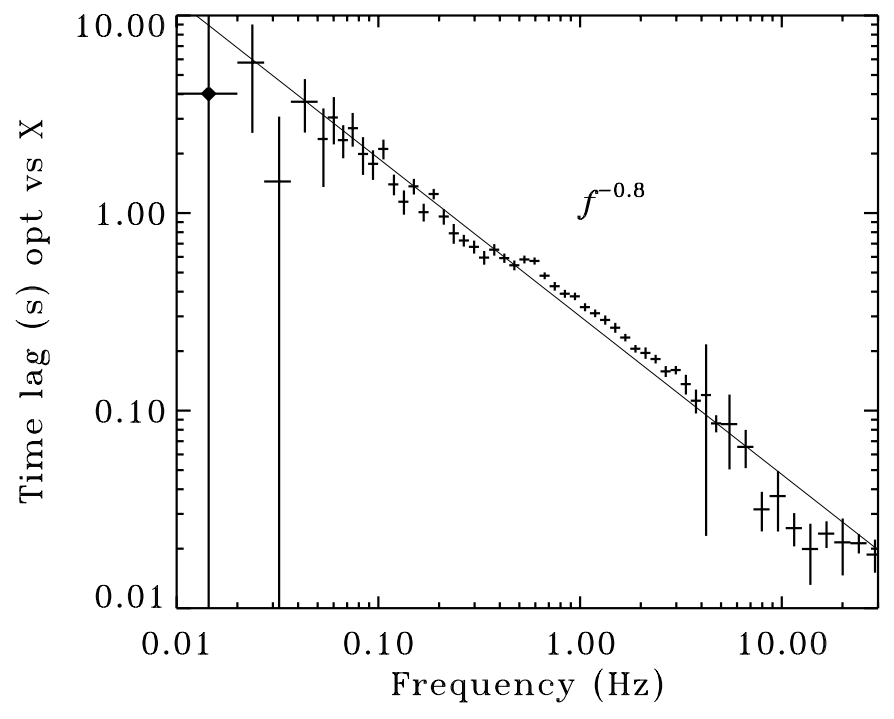

Fig. 8. Frequency dependent optical time-lags. Filled diamonds stand for negative lags.

apparent in the optical X-ray CCF. Indeed, since in the Fourier method, the light curves are decomposed over cosine functions, an anti-correlation at negative optical lags appears exactly in the same way as a positive correlation at positive lags. Thus, the optical dip and peak information are all mixed together in the Fourier representation.

\section{Superposed shot and dip analysis}

Besides Fourier and time domain analysis, there is a third method occasionally used to study the variability of X-ray binaries. It is based on the selection and averaging of flares events in the light curves.

In the following we will apply a method similar to that used by Negoro et al. (2001) to study the average shot profile in Cyg X-1. We select flare events in the lower energy light curve (b1,2-5.9 keV). These shots are selected according to the same criteria as those used by Negoro et al. (2001): the peak count rate of the shot is greater than $f$ times the local count rate as 

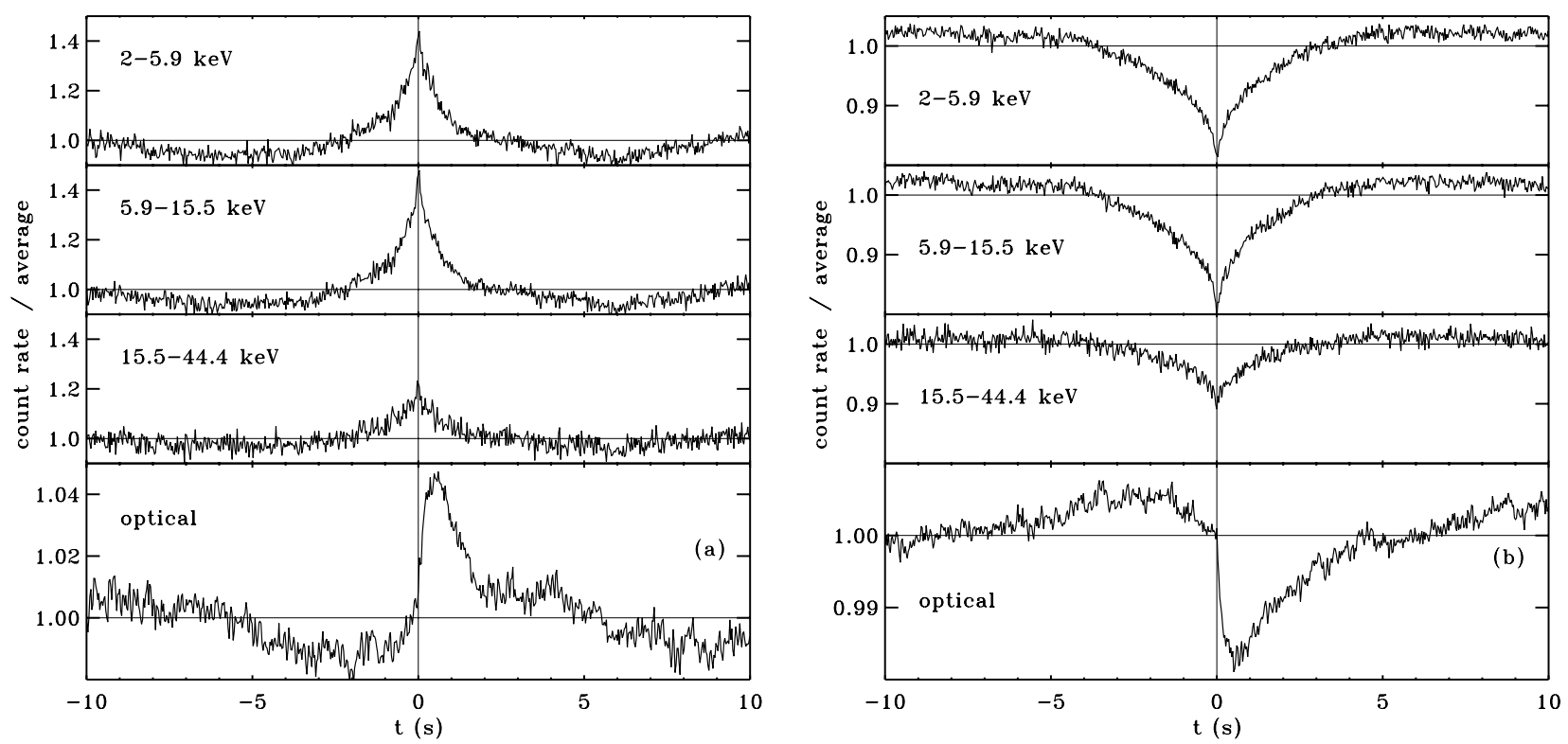

Fig. 9. Results from the shot (panel a)) and dip (panel b)) superposition technique when the flares or dips are selected in band b1. The selection parameters (see Sect. 4) are $f=2, t_{\mathrm{p}}=8 \mathrm{~s}, t_{\mathrm{m}}=32 \mathrm{~s}$ in both panels.

obtained from an average over time $t_{\mathrm{m}}$. The peak bin is further required to have the maximum count-rate over bins within $t_{\mathrm{p}}$ before and after the peak bin. The selected shots are then peak aligned and averaged. The corresponding pieces of light curves in the b2, b3 and optical band are centered on the time bin corresponding to the b1 peak and averaged in the same way.

Figure 9a shows the results for $f=2, t_{\mathrm{p}}=8 \mathrm{~s}$ and $t_{\mathrm{m}}=32 \mathrm{~s}$. The light curves were rebinned on $30 \mathrm{~ms}$ time bins before applying the shot selection. For such parameters the resulting average $\mathrm{X}$-ray shot is slightly asymetric, with a duration of $\sim 10 \mathrm{~s}$. The $\mathrm{b} 2$ and $\mathrm{b} 3$ bands appears to present shots that are similar to that in the $\mathrm{b} 1$ band. This illustrates the high degree of coherence between the different energy bands.

The shot in the b3 band has a lower amplitude than the b1 and b2 shots. This is consistent with the energy dependent power spectrum indicating a lower amplitude of variability in the higher energy band. In addition, this enables us to see in a more direct way the spectral evolution leading to the anti-correlation between X-ray hardness and flux discussed in Sect. 2. The average optical light curve corresponding to the shots is similar in shape to that of the optical/ X-ray CCF. This suggests, as previously noted by Spruit \& Kanbach (2002), that the shape of the CCF is representative of the shape of the optical light curve as a response to a shot event.

Then it is interesting to see whether the optical light curve responds only to X-ray flares or is also correlated to other types of events occuring in the X-ray light curve. We thus performed a similar analysis but instead of flares, we selected dips in the b1 band. The selecting criteria were that the minimum count rate of the dip is lower than $1 / f$ times the local count rate as obtained from an average over $t_{\mathrm{m}}$. The minimum bin is further required to have the minimum count-rate over the bins within $t_{\mathrm{p}}$ on either side.

The results are shown in Fig. 9b. Surprisingly, the answer is that the optical responds to X-ray dips in a similar but inverted way as it responds to the X-ray flares. The optical flux rises a few seconds before the minimum in the X-ray light curve, at $t=0$ it decays abruptly with a minimum half a second after the X-ray dip. The panels (a) and (b) of Fig. 9 are actually very similar but with inverted count-rate axis.

To learn about the response of the X-rays to optical fluctuations we performed a similar shot and dip analysis using the optical band as the selecting light curve. The results are shown in Fig. 10. The X-ray shots and dips are not simply the same as in Fig. 9 shifted by $\sim 0.5 \mathrm{~s}$ as one would expect if the correlation was linked only to the X-ray dips and shots. The X-ray response to optical shots and dips is very asymmetric. The optical shots are associated with an X-ray flux that rises slowly during a few seconds and decays sharply in $\sim 0.5 \mathrm{~s}$. There is an indication for the presence of an X-ray dip after the peak in the optical. A similar asymmetry is apparent in the dip analysis. Thus from Figs. 9 and 10, it appears that the correlated optical and X-ray shots and dips all have a profile resembling that of the optical/X-ray CCF (modulo the relevant symmetries).

The superposition method is far less rigorous than usual time-domain and Fourier techniques. In particular the results are affected by strong biases. It tends to favor a certain range of time-scales and amplitude depending on the selection criteria. In general, the selected events are not representative of the whole variability of the source. It could be also, that the light curves are not made of a superposition of shots at all. Moreover a description of the variability of accreting black hole sources in terms of shots models (e.g. Poutanen \& Fabian 1999 and reference therein) requires, in general, events with a broad range of time-scales and amplitude. The superposed events are not representative of a clearly defined scale, rather, they represent an average over a range of scales that is poorly controlled.

The drawbacks of the method can be taken into our advantage. By changing the selection criteria one can select (in a very qualitative way) the time-scale and amplitude of the 

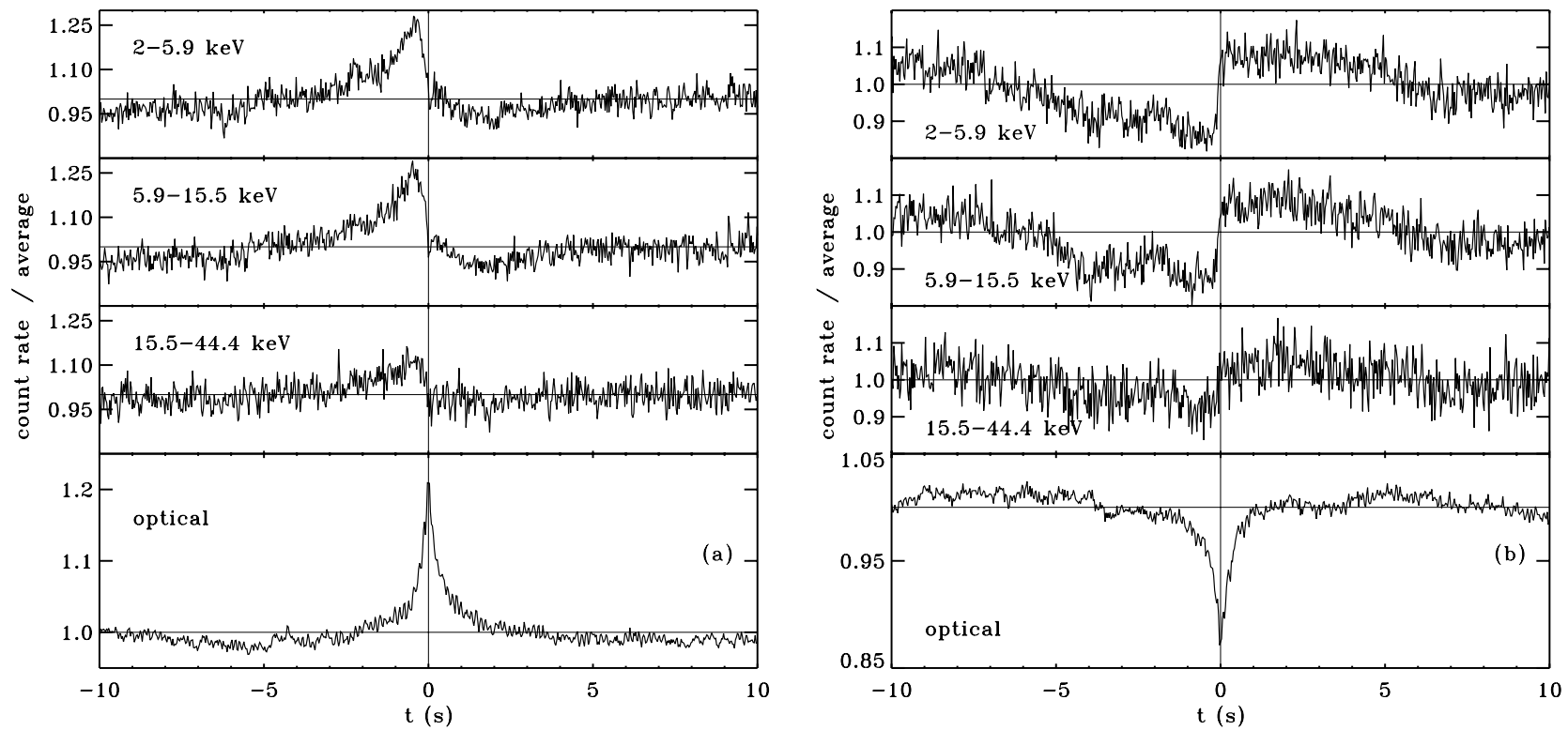

Fig. 10. Results from the shot (panel a)) and dip (panel b)) superposition technique when the flares or dips are selected in the optical band. The selection parameters (see Sect. 4) are $f=1.2, t_{\mathrm{p}}=8 \mathrm{~s} t_{\mathrm{m}}=32 \mathrm{~s}$ in both panels.
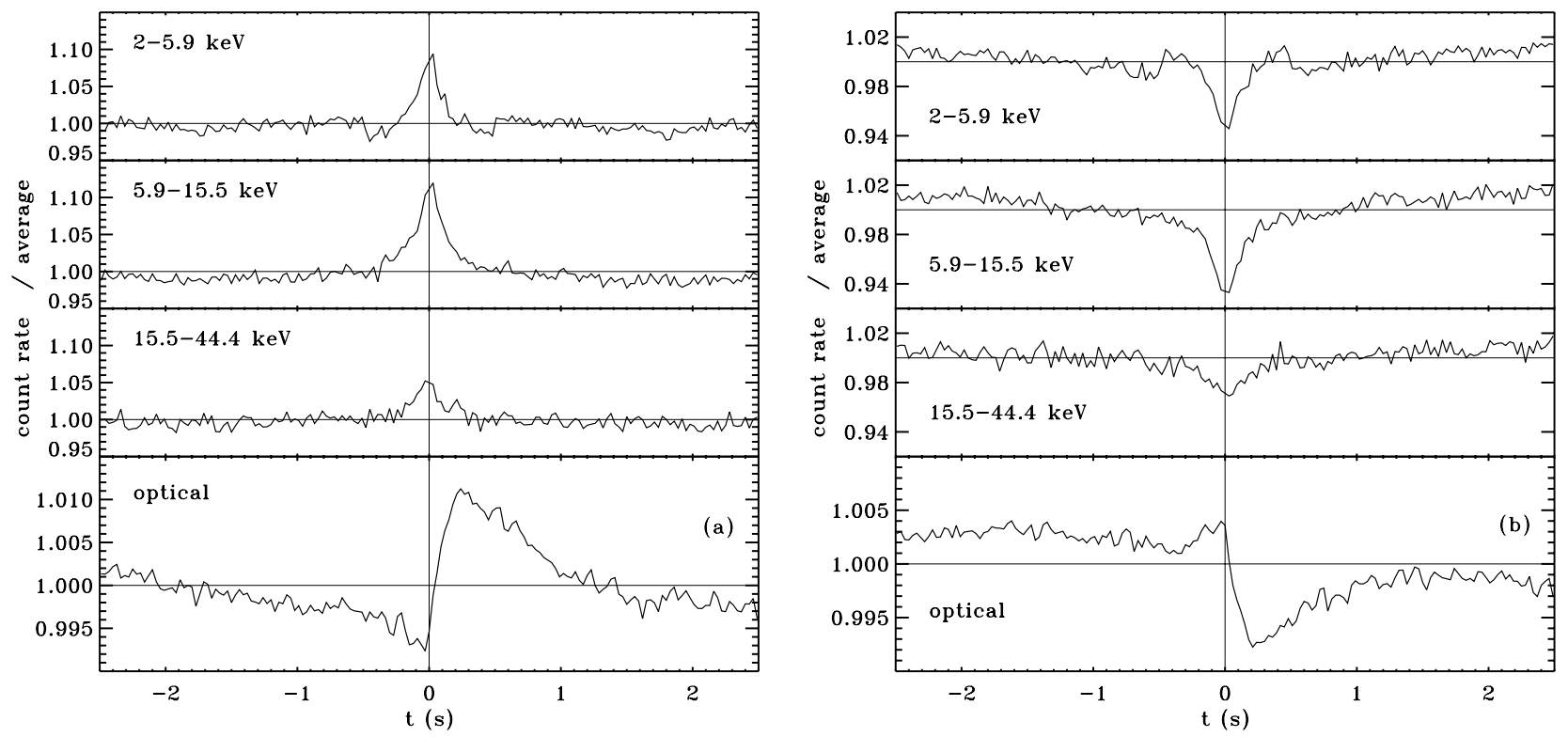

Fig. 11. Results from the shot (panel a)) and dip (panel b)) superposition technique using band b1 as the selecting band. The selection parameters (see Sect. 4) are $f=1.8, t_{\mathrm{p}}=0.5 \mathrm{~s}$ and $t_{\mathrm{m}}=5 \mathrm{~s}$.

typical selected events. For instance, Fig. 11 shows the results of the shot and dip superposition for $t_{\mathrm{p}}=0.5 \mathrm{~s}$ and $t_{\mathrm{m}}=5 \mathrm{~s}$ and $f=1.8$ selected in the b1 band. The resulting average profiles have clearly a lower amplitude and shorter duration $(<1) \mathrm{s}$. The interesting thing is that the both the X-ray and optical light curves look like a rescaled version of Fig. 9. In particular, the softening of the spectrum with the enhanced count rate, and the presence of an optical dip and flare respectively before and after the X-ray peak. The superposition analysis thus confirms that both the optical pre-dip and post-peak are present over a wide range of time-scales.
More importantly, the superposition method, despite all its biases, reveals that the optical/X-ray correlation is not triggered by a single type of event (flare or dip) in the light curves. The correlation is thus a global property of the light curves. Flares and dips of different shapes and time-scales appear to contribute to the shape of the total optical X-ray CCF. Strikingly, they all contribute in a similar way to the formation of the predip and post-peak features.

Also, when comparing the superposed light curves we see that the changes occur on similar time-scales, and almost at the same time in the different bands. The general impression is that high (low) optical fluxes are associated with a sharp 
decay (rise) in the X-rays. In other words the optical appears to be proportional to the opposite of the time derivative of the $\mathrm{X}$-rays. If such a relation was to hold strictly, we would expect a constant optical phase-lag of value $\pi / 2$ (due to the fact that a derivation in the time domain translates into a constant phase factor in the Fourier domain). In contrast, we found (see Fig. 5) that the phase-lags do depend on Fourier frequency and approximatively cover the range $0-\pi$. Nevertheless, we see that the phase lags are close to $\pi / 2$ in the frequency range $0.1-1 \mathrm{~Hz}$ where the coherence is significant. Therefore, in the frequency range where most of the correlation occurs, the optical is indeed related to the time derivative of the correlated X-ray flux. Equivalently, the $\sim \pi / 2$ lags can be interpreted as the X-ray light curve scaling like the time derivative of its correlated optical counterpart. Due to the remarkable symmetry of the correlation, this interpretation would be also roughly consistent with the general trend observed in the superposition analysis (Figs. 9-11). There is thus a "differential" relation between the optical and the X-rays: the variability of a light curve appears related to the time derivative of the correlated fraction of the other.

With this regard, rather than using concepts like "precognition" dip and delays, the shape of the superposed light curves and CCF could be interpreted as the signature of a process affecting at the same time, both the X-ray and optical emitting region. In this context, it is not clear whether the "differential" relation reflects an intrinsic "differential" property of the underlying physical process, or on the contrary, emerges casually from the fact that the phase-lags are close to $\pi / 2$ in the frequency range where coherence is important.

\section{Discussion}

Several studies already emphasized the similarity of the X-ray power spectrum in XTE J1118+480 with that of typical black hole candidates in the hard state. Here we find that the similarity extends to other X-ray timing features, namely:

- The weak energy dependence of the power spectrum.

- The reduction of the integrated rms amplitude at high energy.

- The shape of the X-ray ACF and CCFs.

- The presence of an anti-correlation between the X-ray flux and hardness.

- The unity coherence between X-ray bands over a wide range of frequencies.

Together with the presence of striking spectral similarities (Frontera et al. 2001, 2003), this probably makes XTE J1118+480 a typical hard state source. On the other hand our study suggests that XTE J1118+480 might differ from typical hard states sources by the presence of soft lags at frequencies below $0.1 \mathrm{~Hz}$ apparent both in the Fourier analysis and through the asymmetry found in the X-ray CCFs.

Our study of the correlated optical/X-ray flickering reveals that:

- The optical X-ray CCF is independent of the X-ray band.
- The optical band is correlated not only to the X-ray flux but also, and in a similar way, to the rapid X-ray spectral fluctuations. This is shown by the computation of the optical flux vs. X-ray hardness CCF and the superposition analysis.

- The optical phase-lags increase significantly with Fourier frequency covering the range $0-\pi$. This leads to time-lags decreasing roughly like $f^{-0.8}$.

- The optical/X-ray coherence is maximum $(\sim 0.3)$ for fluctuations in the range $1-10 \mathrm{~s}$ and decreases sharply both at shorter and longer time-scales.

- The shape of the X-ray CCFs asymmetry (Fig. 2) is reminiscent of the asymmetry of the optical/X-ray CCF (Fig. 3a). This may indicate a connection between the $\mathrm{X}$-ray and optical lags. This suggestion is strengthened by the dependence of the optical time-lags at higher frequency $\sim f^{-0.8}$ that is comparable to the dependence of the $\mathrm{X}$-ray lags in hard state black holes.

- The filtered CCF analysis as well as the superposition technique shows that the underlying process, giving rise to the correlated variability, acts in a similar but rescaled way at different time- and amplitude-scales of the fluctuations.

- The superposition technique shows that the optical response is not triggered by a specific type of event in the X-ray light curve (shot or dip). Rather, events of very different shapes and time-scales contribute in a similar way to the shape of the optical/X CCF. In the range of frequencies where the optical/X-ray coherence is maximum, the light curve in one band is related to the time derivative of its correlated counterpart.

One of the main result of this work is that the optical/X-ray correlation can be described as self-similar. We note that this scaling in time is consistent with what SK02 find by the completely independent method of principal component analysis (PCA). With this analysis, the X-ray/optical cross correlation is computed on short segments of data (25s long), yielding a large number of samples (300) of the cross correlation, which turn out to be somewhat variable in shape. With the PCA (e.g. Kendall 1980), the similarities and difference between samples are analyzed by finding linear combinations of the samples which are statistically uncorrelated. If the variability of the signal comes about as a mixture of a few signals of fixed shape but independently varying amplitudes, the PCA will extract these shapes as the "principal components". In SK02, two significant components were found, both of which were of the dip-plusspike shape but on time scales differing by a factor 3 . This is similar to the results presented here (compare Fig. 9 lower left with Fig. 11 lower left panel). The "scaling" of the components suggests that there is just one underlying process, but that it can take place on different time scales, possibly in a continuous fashion. The filtered CCF analysis (see Fig. 4) and the power-law dependence of optical time-lag on Fourier frequency (Fig. 8), suggest that indeed, this underlying process is continuously self-similar. Actually, the PCA is not well suited to study such a continuous variations. If the signal shape varies in a continuous way, the PCA will represent this continuum by a number of discrete components. These components will be representative of the range of variation through this continuum. 
Moreover, the PCA is sensitive to the coherence and amplitude of variability of the two signals at a given time-scale. Only the time-scales where both the coherence and the amplitude of variability is high will contribute significantly to the variability of the CCF. The finding of SK02 that there are only two significant components simply reflects the fact that the coherence is high only in a relatively narrow range of Fourier frequencies. In other words, the shape of CCF at different time-scales appears to be continuously self-similar, but the amplitude of the average $\mathrm{CCF}$ is dominated by the two time-scales evidenced by the PCA of SK02 and our shot selection analysis.

This scaling is not readily explained by the current accretion models. The ideas that were proposed so far to explain the long optical lags were based on the propagation time between two distinct $\mathrm{X}$-ray and optical emitting regions that are causally connected. In the reprocessing models the lags are interpreted as the light travel-time between the corona and the disc. In the Kanbach et al. (2001) scenario a perturbation propagates from the X-ray corona to the optical outflow photosphere. In such propagation models, the nearly scale-invariant correlation then requires that the small-scale fluctuations have a large propagation speed (outflow model) or alternatively that there are numerous pairs of X-ray/optical emitting regions at different distances from each other, the small-scale perturbation being produced in the closest pairs. Propagation models thus appear strongly constrained and require some complications to fit the scale-invariance. Also as discussed in K01 the propagations models encounter important difficulties in producing features such as a precognition dip that occurs together with a post-peak.

Although the lags indicate a form of causal connection between the X-rays and optical emitting regions they do not necessarily involve a propagation process. Rather, the event superposition analysis suggests that the complicated correlation could be the signature for a self-similar process acting simultaneously in both emitting regions with different manifestations in both bands.

The apparent advances/delays of the optical signal with respect to the X-rays could then be a consequence of a (somewhat complex) evolution of the spectral shape of the emitted radiation during an X-ray event. Such an explanation has already been proposed for the lags observed between hard and soft X-rays in Cyg X-1 (e.g. Poutanen \& Fabian 1999). In this case the hard and soft $\mathrm{X}$-rays are be produced by a single emission mechanism and in the same physical volume. In our case however the situation is more complex. The optical/X CCF is much more complex than the X-ray CCFs and the emission process in the two band is probably different. It is also not clear whether the X-ray and optical emission are produced in the same physical region. Finding a consistent physical scenario that would make such a model predictive and testable is very challenging.

In the jet model of Markoff et al. (2001), the X-rays and optical are both formed through synchrotron emission in the jet. The X-ray CCF could be, in principle due to a characteristic time-evolution of of the distributions of the emitting particles in the jet. The difficulty, here, is that no obvious physical mechanism can produce such a spectral evolution.
In other models, such as the ADAF model of Esin et al. (2001) or the coronal model of Merloni et al. (2000) both the optical and X-rays are emitted in the hot inner part of the accretion disc. For instance, if we assume that the optical is selfabsorbed synchrotron radiation produced by the Comptonising cloud itself (with a temperature $k T_{\mathrm{e}} \sim 100 \mathrm{keV}$ as estimated by Frontera et al. 2001, 2003). Then, the observed optical flux implies a size of the emitting region of a few hundred Schwarzschild radii. Incidentally, this dimension is of the same order as the cold disc inner radius estimated in XTE J1118+480 from fits of the optical to EUV spectra (see Chaty et al. 2003). Then the correlated variability could be caused, for instance, by temporal fluctuations of the magnetic field and/or optical depth of the comptonising plasma. The details are however to be precised and physically motivated. This scenario also encounter a problem to reproduce the observed optical spectrum. This spectrum indeed appears much flatter than that produced through self-absorbed synchrotron. Thus, again, some complications (e.g. strong gradients of magnetic field and/or plasma temperature of the Comptonising plasma) would be required to fit the spectral data.

It could be also that, contrary to what is assumed in the above mentioned models, the X-ray and optical are produced in two distinct regions (e.g. disc+jet). In this case a spectral evolution scenario could also, in principle, account for the correlated X-ray optical variability. Then, the two regions should be physically connected on a time-scale shorter than $\sim 0.1 \mathrm{~s}$, that is the shortest time-scale on which we have been able to observe the typical spectral evolution. The complication with such a model is that it should provide a physical description of the connection between the two regions that is well beyond the simple radiation physics. In the case where the X-rays come from the disc and the optical from the jet, the optical X-ray CCFs, their self-similarity and the apparent "differential" relation between the two light curves would constitute important signatures of the underlying accretion-ejection process.

Acknowledgements. This work was partly supported by the European Commission (contract number ERBFMRX-CT98-0195, TMR network "Accretion onto black holes, compact stars and protostars"). JM also acknowledges fundings from the MURST (COFIN98-02-15-41) and PPARC. We thank Andrea Merloni for valuable discussion.

\section{References}

Chaty, S., Haswell, C. A., Malzac, J., et al. 2003, MNRAS, submitted Dubus, G., Kim, R., Menous, K., Szkody, P., \& Bowen, D. 2001, ApJ, 553, 307

Esin, A. A., McClintock, J. E., Drake, J. J., et al. 2001, ApJ, 555, 483

Fabian, A. C., Guilbert, P. W., Motch, C., et al. 1982, A\&A, 111, L9

Fender, R. P., Hjellming, R. M., Tilanus, R. P. J., et al. 2001, MNRAS, 322, L23

Frontera, F., Zdziarski, A. A., Amati, L., et al. 2001, ApJ, 561, 1006

Frontera, F., Amati, L., Zdziarski, A. A., et al. 2003, ApJ, in press [astro-ph/0304261]

Feng, Y. X., Li, T. P., \& Chen, L. 1999, ApJ, 514, 373

Garcia, M., Brown, W., Pahre, M., et al. 2000, IAU Circ., 7392, 2

Georganopoulos, M., Aharonian, F. A., \& Kirk, J. G. 2002, A\&A, 388, L25 
Haswell, C. A., Skillman, D., Patterson, J., Hynes, R. I., \& Cui, W. 2000, IAU Circ., 7427, 1

Hynes, R. I., Mauche, C. W., Haswell, C. A., et al. 2000, ApJ, 539, L37

Hynes, R. I., Haswell, C. A., Cui, W., et al. 2003, MNRAS, in press [astro-ph/0306626]

Kendall, M. S. 1980, Multivariate analysis, 2nd edition (London: Griffin)

Kanbach, G., Straubmeier, C., Spruit, H. C., \& Belloni, T. 2001, Nature, 414, 180

Li, T. P., Feng, Y. X., \& Chen, L. 1999, ApJ, 521, 789

Maccarone, T. J., Coppi, P. S., \& Poutanen, J. 2000, ApJ, 537, L107

Markoff, S., Falcke, H., \& Fender, R. 2001, A\&A, 372, L25

Mauche, C., Hynes, R., Charles, P., \& Haswell, C. 2000, IAU Circ., 7401, 2

McClintock, J. E., Garcia, M. R., Caldwell, N., et al. 2001, ApJ, 551, L147

McClintock, J. E., Haswell, C. A., Garcia, M. R., et al. 2001, ApJ, 555,447
Merloni, A., Di Matteo, T., \& Fabian, A. C. 2000, MNRAS, 318, L15

Motch, C., Ilovaisky, S. A., \& Chevalier, C. 1982, A\&A, 109, L1

Negoro, H., Kitamoto, S., \& Mineshige, S. 2001, ApJ, 554, 528

Nowak, M. A., Vaughan, B. A., Wilms, J., Dove, J. B., \& Begelman, M. C. 1999, ApJ, 510, 874

Poutanen, J., \& Fabian, A. C. 1999, MNRAS, 306, L31

Remillard, R., Morgan, E., Smith, D., \& Smith, E. 2000, IAU Circ., 7389, 2

Revnivtsev, M., Sunyaev, R., \& Borozdin, K. 2000, A\&A, 361, L37

Spruit, H. C., \& Kanbach, G. 2002, A\&A, 391, 225

Straubmeier, C., Kanbach, G., \& Schrey, F. 2001, Exp. Astron., 11, 157

Vaughan, B. A., \& Nowak, M. A. 1997, ApJ, 474, L43

Wagner, R. M., Foltz, C. B., Shahbaz, T., et al. 2001, ApJ, 556, 42

Wood, K. S., Ray, P. S., Bandyopadhyay, R. M., et al. 2000, ApJ, 544, L45

Yamaoka, K., Ueda, Y., Dotani, T., Durouchoux, P., \& Rodriguez, J. 2000, IAU Circ., 7427, 2 